“(C) 2018 IEEE. Personal use of this material is permitted. Permission from IEEE must be obtained for all other uses, in any current or future media, including reprinting/republishing this material for advertising or promotional purposes, creating new collective works, for resale or redistribution to servers or lists, or reuse of any copyrighted component of this work in other works." 


\title{
Compact, Low-Profile, Bandwidth-Enhanced Substrate Integrated Waveguide Filtenna
}

\author{
Kun-Zhi Hu, Ming-Chun Tang, Senior Member, IEEE, Mei Li, Member, IEEE, \\ and Richard W. Ziolkowski, Fellow, IEEE
}

\begin{abstract}
In this letter, a compact, low-profile, bandwidth enhanced, dual-cavity substrate integrated waveguide (SIW) filtenna is demonstrated. Two SIW cavities are stacked vertically on top of each other. A complementary split ring resonator (CSRR) slot is etched in the top surface of the uppermost cavity, causing the top surface to act as a patch antenna. The operational impedance bandwidth is significantly enhanced by merging the three resonances that arise from this configuration. One is introduced by the patch and the other two are inherently generated by the two cavities. A metallized coupling post is introduced from the ground plane through both cavities to the upper surface to excite the fundamental resonant mode of the patch, as well as to electromagnetically couple the two cavities. The optimized filtenna was fabricated by standard printed circuit board (PCB) technology and tested. It has a low profile $0.03 \lambda_{0}$ and compact size $0.62 \lambda_{0} \times 0.62 \lambda_{0}$ at its center frequency, $f_{0}=2.95 \mathrm{GHz}$. The measured results agree well with their simulated values. They demonstrate a $6.3 \%$ fractional bandwidth, a maximum realized gain of $6.73 \mathrm{dBi}$, a flat gain profile within its passband, and excellent out-of-band selectivity.
\end{abstract}

Index Terms - Bandwidth, compact antenna, filtenna, low-profile antenna, patch antenna, substrate integrated waveguide (SIW)

\section{INTRODUCTION}

D riven by the increasing demand for smaller, cheaper wireless communications and sensor devices, platform miniaturization has become a key consideration. This trend requires size reductions in both the radio frequency (RF) antennas and the associated front-end circuits. While progress in printed-circuit board (PCB) technologies and electrically small antennas has occurred, the use of multifunctional components has proven to be another effective methodology to

Manuscript received on May 25, 2018; revised on 5 July, 2018; and accepted on 7 July, 2018. This work was supported in part by the Graduate Scientific Research and Innovation Foundation of Chongqing, China contract number CYS18062, in part by the National Natural Science Foundation of China contract numbers 61471072 and 61701052 , in part by the Funding of the leading research talent cultivation plan of Chongqing University contract number cqu2017hbrc1 A08, in part by the Fundamental Research Funds for the Central Universities contract number 2018CDQYTX0025, in part by Opening subject of State Key Laboratory of Millimeter Waves contract number K201732, and in part by the Australian Research Council grant number DP160102219. (Corresponding author: Ming-Chun Tang.)

K. -Z. Hu, M. -C. Tang, and M. Li are with the Key Laboratory of Dependable Service Computing in Cyber Physical Society Ministry of Education, College of Communication Engineering, Chongqing University, Chongqing 400044, China and with the State Key Laboratory of Millimeter Waves, Nanjing, 210096, China (E-mail: tangmingchun@cqu.edu.cn);

R. W. Ziolkowski is with the Global Big Data Technologies Centre, University of Technology Sydney, Ultimo NSW 2007, Australia (E-mail: Richard.Ziolkowski@uts.edu.au). realize both size reductions and performance improvements of the whole system. This approach has led to the development of filtennas, a multifunctional component that combines both filtering and radiation performance characteristics into a single module, and thus reduces the dimensions and cost of RF front-ends.

Because they possess the advantages of low loss, low cost, high quality factors, and easy integration with other planar circuits [1-6], substrate integrated waveguide (SIW) technologies have become popular and widely used in antenna and filter applications. Nevertheless, the SIW-based filtennas developed to date have seen only limited use because they have narrow bandwidths. The SIW-based filtenna reported in [7] was constructed with two resonant cavities. One cavity includes a slot radiator while also acting as a component of the filter. The reported fractional bandwidth (FBW) was only $1.56 \%$. In [8], a filter synthesis approach was adopted to design a filtenna based on two vertically stacked cavities. A 3.0\% FBW was achieved.

Several methods have been reported to increase the bandwidths of SIW-based filtennas. In particular, a common approach is to introduce more cavities, cascading them either vertically or horizontally, and, hence, introducing more overlapping resonance frequencies. For example, the filtenna presented in [9] obtained a 5.5\% FBW by using three cavities integrated vertically. The last cavity in that design also acted as a cavity-backed slot antenna. Similarly, the filtenna reported in [10] used four cascaded horizontal cavities. It achieved a wider, $6.0 \%$ FBW. Nevertheless, simply increasing the total number of cavities to achieve more bandwidth inescapably suffers from limitations. Notably, it introduces relatively high profiles (vertical stack) or large transverse dimensions (horizontal cascade), and thus leads to increasingly complex designs and larger front-end volumes.

In this letter, a novel compact, low-profile, bandwidth-enhanced, SIW-based filtenna is reported. Two SIW cavities are stacked vertically. Instead of using the more common cavity-backed rectangular slot as the radiator [7-10], a complementary split ring resonator (CSRR) is etched on the top surface of the top SIW cavity. This top surface then acts as a CSRR-slot modified patch radiator. A circular post is introduced that traverses both cavities to facilitate electromagnetic (EM) coupling between them and to directly excite the patch. By merging the additional resonance arising from this modified patch with the two resonances generated by the two cavities, the filtenna's bandwidth is significantly improved without an increase of its overall dimensions. The measured results demonstrate that this dual-cavity filtenna with 
dimensions: $0.62 \lambda_{0} \times 0.62 \lambda_{0} \times 0.03 \lambda_{0}$, achieved a $6.3 \%$ FBW along with good radiation performance and out-of-band selectivity.

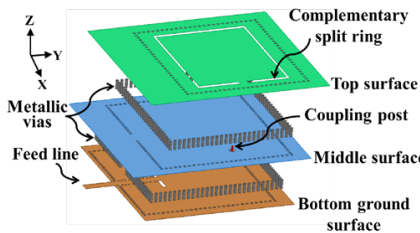

(a)

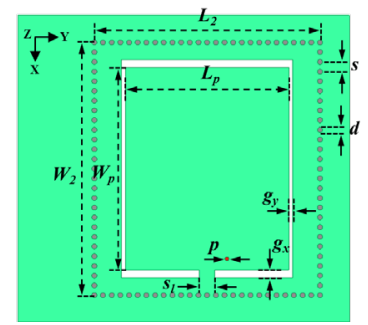

(c)

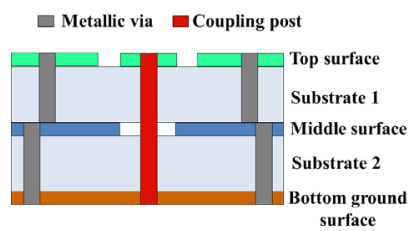

(b)

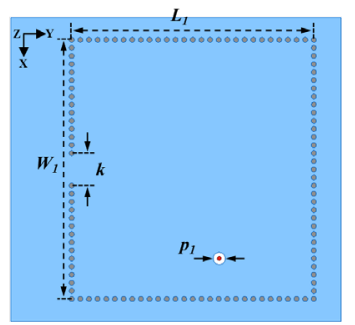

(d)

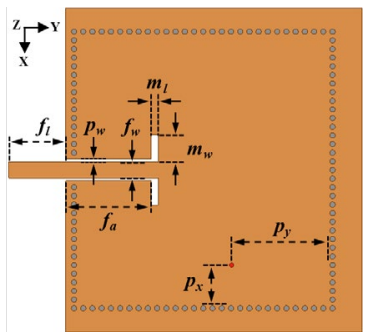

(e)

Fig. 1 Dual-cavity SIW-based filtenna. (a) 3-D view. (b) Side view. (c) Top surface. (d) Middle surface. (e) Bottom ground surface.

\section{Filtenna Configuration}

The dual-cavity SIW-based filtenna configuration is shown in Fig. 1 along with its design parameters. The filtenna is fabricated with two pieces of Taconic TLY-5 copper-cladded substrate with a thickness of $1.575 \mathrm{~mm}$, a relative dielectric constant of 2.2, and a loss tangent of 0.0009 . The sidewalls of each cavity are formed by a row of metallic vias, each via having the diameter $d$ and being spaced equally apart by a center-to-center distance $s$. A CSRR slot is etched into the top metallic surface. A metallized circular post is introduced to directly connect the CSRR-slot modified patch with the bottom ground surface. A small circular slot with diameter $P_{l}$ that is concentric with the post is etched into the middle metallic surface to avoid shorting the post. A $50-\Omega$ grounded coplanar waveguide $(\mathrm{GCPW})$ is adopted as the feed element that directly excites the bottom SIW cavity. Two rectangular slots are etched into the bottom ground surface, being arranged symmetrically on the two sides of GCPW, to improve the impedance matching. A microstrip line is added to one side of the GCPW, extending from it to off the substrate. It acts as an impedance transformer. Optimization of the design was performed with the Ansoft High Frequency Structure Simulator (HFSS). The optimized design parameters are listed in Table I. Photographs of the fabricated prototype are shown in Fig. 2. Copper screws were adopted to tightly fasten the two substrates together. A 50- $\Omega$ SMA connector was mounted for coaxial feeding of the GCPW.
TABle I. Dual-CAVity SIW-Based Filtenna Design Parameters (ALL DIMENSIONS ARE IN MILLIMETERS)

\begin{tabular}{|c|c|c|c|}
\hline$L_{1}=47.6$ & $L_{2}=44.2$ & $L_{p}=32.0$ & $g_{x}=5.0$ \\
\hline$W_{l}=51.2$ & $W_{2}=49.4$ & $W_{p}=39.6$ & $g_{y}=1.5$ \\
\hline$s=1.7$ & $d=1.0$ & $p=0.6$ & $p_{1}=2.2$ \\
\hline$s_{l}=3.0$ & $k=5.4$ & $m_{l}=1.3$ & $m_{w}=5.0$ \\
\hline$p_{w}=0.5$ & $f_{l}=10.5$ & $f_{w}=3.0$ & $f_{a}=15.7$ \\
\hline$p_{x}=7.5$ & $p_{y}=18.1$ & \multicolumn{2}{|c|}{ Null } \\
\hline
\end{tabular}

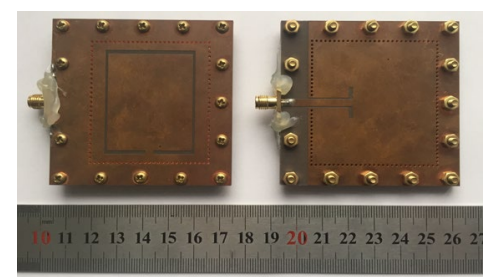

Fig. 2 Photograph of the fabricated dual-cavity SIW-based filtenna. Left: top view. Right: bottom view.

\section{OPERATIONAL MECHANISMS}

The operational mechanisms of the prototype filtenna were investigated extensively. A dual-cavity filter was designed initially and its transmission response was analyzed with the aid of an equivalent circuit model. The performance characteristics of our prototype CSRR-based filtenna were then investigated with this design methodology.

\section{A. Dual-Cavity Filter Design}

A two-pole cavity bandpass filter with Chebyshev frequency response was designed according to known filter synthesis techniques [11]. The equivalent circuit model of this two-pole cavity filter and its design parameters are presented in Fig. 3.

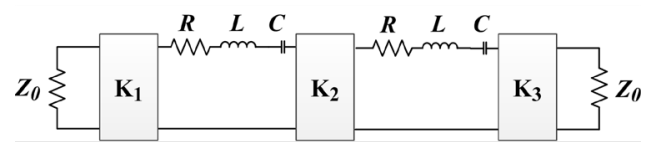

(a)

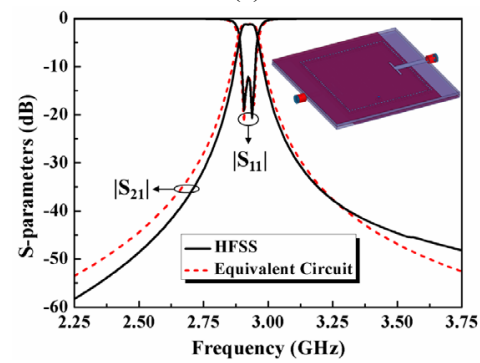

(b)

Fig. 3 (a) Equivalent circuit model of the two-pole cavity filter with $\mathrm{K}_{1}=\mathrm{K}_{3}=$ $2.3 \Omega, \mathrm{K}_{2}=0.14 \Omega, R=0.02 \Omega, L=0.373 \mathrm{nH}, C=7.934 \mathrm{pF}$. (b) Comparison of HFSS and equivalent circuit simulated S-parameters.

The coupling matrix of this filter is represented as

$$
M=\left(\begin{array}{ccccc} 
& S & 1 & 2 & L \\
S & 0 & 0.9291 & 0 & 0 \\
1 & 0.9291 & 0 & 1.1159 & 0 \\
2 & 0 & 1.1159 & 0 & 0.9291 \\
L & 0 & 0 & 0.9291 & 0
\end{array}\right)
$$

The corresponding internal coupling coefficient and external quality factors are obtained from this coupling matrix by using the formulas [11]: 


$$
k_{12}=\mathrm{FBW} \cdot M_{12} \quad Q_{e}=\frac{1}{\mathrm{FBW} \cdot M_{S 1}^{2}}
$$

where $M_{12}$ and $M_{\mathrm{S} 1}$ are, respectively, the generalized coupling coefficients between the two cavities, and between the cavity and the source; and where FBW is the fractional bandwidth. Applying them to (1), one obtains $k_{12}=0.02$ and $Q_{\mathrm{e}}=64.3$. To realize this value of $k_{12}$ between the bottom and top cavities in our design, a metallized post that traverses both of them was employed. The coupling strength is advantageously adjusted simply by changing its position when the diameter of the post is fixed. It is then extracted from full-wave simulations of the filter with the formula [11]:

$$
k_{12}=\frac{f_{p 2}^{2}-f_{p 1}^{2}}{f_{p 2}^{2}+f_{p 1}^{2}}
$$

where $f_{p 1}$ and $f_{p 2}$ are, respectively, the lower and higher resonance frequencies obtained from a corresponding eigenmode simulation of the two cavities. The external coupling is facilitated by connecting the GCPW to the input/output ports of the filter. Its coupling strength is controlled mainly by the length of the GCPW coupling slots. The external quality factor $Q_{\mathrm{e}}$ is also extracted from the simulations using [11]

$$
Q_{e}=\frac{2 \pi f_{0} \cdot \tau}{4}
$$

where $f_{0}$ is the resonance frequency and $\tau$ is the corresponding group delay. The values of the inverter parameters in the circuit model: $\mathrm{K}_{1}, \mathrm{~K}_{2}$ and $\mathrm{K}_{3}$ can then be evaluated with (3) and (4) as:

$$
\mathrm{K}_{1}^{2}=\mathrm{K}_{3}^{2}=\frac{Z_{0} \cdot 2 \pi f_{0} \cdot L}{Q_{e}} \quad \mathrm{~K}_{2}=k_{12} \cdot 2 \pi f_{0} \cdot L
$$

The simulated S-parameters obtained with HFSS and the equivalent circuit model, are given in Fig. 3. They are in good agreement and demonstrate stable bandpass performance and good out-of-band rejection. Since there are only two transmission poles within the passband, the operational bandwidth is relatively narrow. The center frequency and FBW values are $2.92 \mathrm{GHz}$ and $1.8 \%$, respectively.

\section{B. Design of CSRR-based Filtenna}

As illustrated in Fig. 1, the CSRR is etched on the top surface of the upper SIW cavity symmetrically with respect to the center axis of the upper cavity. It has the length $W_{p}$ and width $L_{p}$. Their values in Table I cause the CSRR-modified patch to resonate around $2.92 \mathrm{GHz}$. Excitation of the fundamental modes of both cavities and the modified patch are facilitated by the optimized position of the post. Moreover, the post directly connects the top and bottom metallic surfaces in order to excite the CSRR-modified patch effectively and, hence, produce the resonant mode of the patch.

The equivalent circuit model of the CSRR-based filtenna is shown in Fig. 4(a). The two vertically cascaded cavities are modeled as the RLC resonators labeled with the parameters: resistance $R_{i}$ inductance $L_{i}$ and capacitance $C_{i}(i=1,2)$. The impedance inverters are marked again as $\mathrm{K}_{1}, \mathrm{~K}_{2}$ and $\mathrm{K}_{3}$. They again represent the couplings between the source and the lower cavity, between the two cavities, and between the patch and the upper cavity, respectively. In contrast to the equivalent circuit model in Fig. 3, the CSRR-modified patch radiator is modeled as the RLC series-resonant circuit with $R_{p}, L_{p}$ and $C_{p}$. Since the presence of CSRR has an effect on the couplings between the two cavities and between the patch and upper cavities, the values of $\mathrm{K}_{1}, \mathrm{~K}_{2}$ and $\mathrm{K}_{3}$ are different from those in Fig. 3. Additionally, the HFSS simulated results of the filtenna with the traditional rectangular coupling slot (e.g., [8]-[10]) etched along the $x$-axis in the top surface of the upper cavity and with its center on the $y$-axis and displaced along it from the cavity center by $3.65 \mathrm{~mm}$, but with the same height $\left(0.03 \lambda_{0}\right)$, is presented in Fig. 4(b) for comparison. It has a FBW $=1.8 \%$, which is one third the FBW of our prototype.

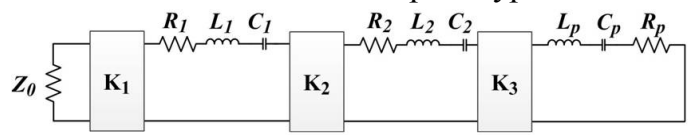

(a)

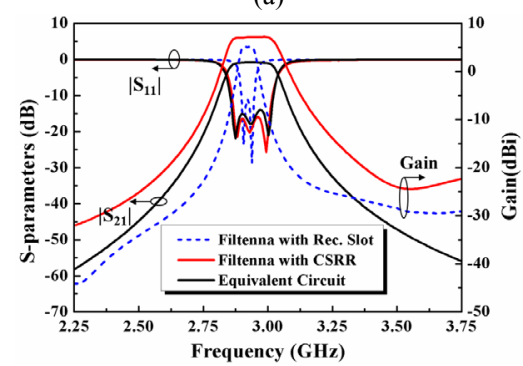

(b)

Fig. 4 (a) Equivalent circuit model of the CSRR-based filtenna with $Z_{0}=50 \Omega$, $R_{1}=0.03 \Omega, L_{1}=0.383 \mathrm{nH}, C_{1}=7.7085 \mathrm{pF}, R_{2}=0.022 \Omega, L_{2}=0.353 \mathrm{nH}, C_{2}=$ $8.3162 \mathrm{pF}, \mathrm{K}_{1}=3.9889 \Omega, \mathrm{K}_{2}=0.3137 \Omega, \mathrm{K}_{3}=0.2639 \Omega, R_{p}=0.289 \Omega, L_{p}=$ $0.309 \mathrm{nH}, C_{p}=9.4772 \mathrm{pF}$. (b) Simulated results of different models.

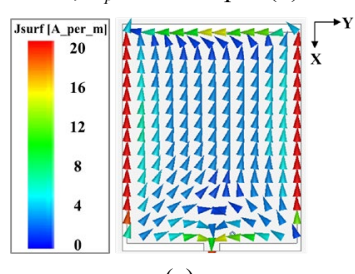

(a)

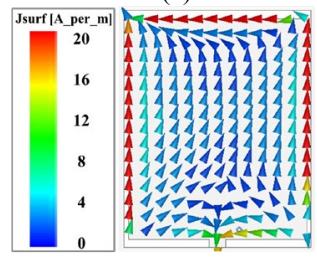

(c)

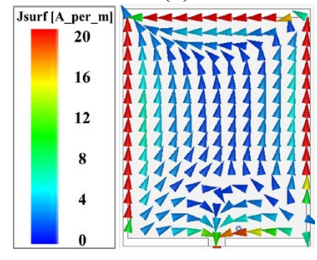

(e)

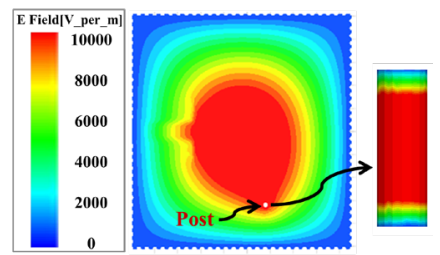

(b)

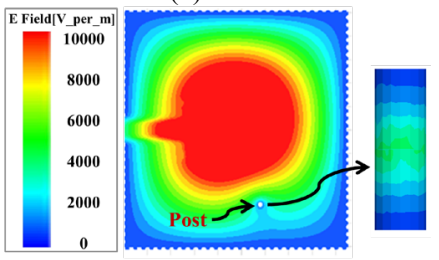

(d)

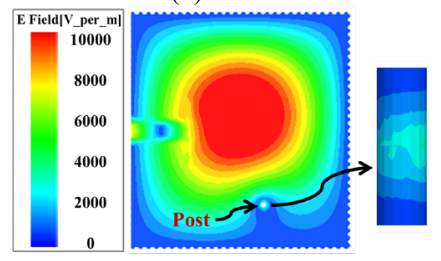

(f)

Fig. 5 Current distribution on the patch and electric field distribution on the bottom XY-surface at (a), (b) $2.88 \mathrm{GHz}$; (c), (d) $2.93 \mathrm{GHz}$; and (e), (f) 2.99 $\mathrm{GHz}$.

The current and electric field behaviors of our CSSR-based filtenna are illustrated in Fig. 5 for three frequencies: 2.88, 2.93, and $2.99 \mathrm{GHz}$. The current distributions on the radiating patch are plotted in Figs. 5 (a), (c), and (e), respectively. The current distributions are quite similar with each other; this explains the uniform radiation performance over the entire operational band. The electric field distributions on the bottom surface of the 
lower cavity which includes the presence of the post are given in Figs. 5 (b), (d), and (f), respectively. It is noted that the electric field distributions around and on the post in Figs. 5(d) and 5(f) are not as strong as those in Fig. 5(b). This behavior corresponds to the fact that the lowest resonant mode is introduced by the CSRR-modified patch-post combination, and that the other two resonant modes are generated by the two SIW cavities, respectively. This explanation was confirmed further with parametric studies of the post location $p y$ and the CSRR length $W_{p}$. When $p y$ decreases, the first resonance remains stationary while the two cavity resonances separate further. This behavior occurs because the coupling strength between the two cavities is modified. A corresponding modification of the $\mathrm{K}_{2}$ inverter value in the equivalent circuit model in Fig. 4 confirms this behavior. The CSRR-modified patch and post combination controls the first resonance, as confirmed by increasing $W_{p}$ until it appears. The radiation patterns remain basically unchanged as the three resonances are optimized.

\section{Simulated AND MeAsured Results}

A comparison of the simulated and measured reflection coefficients is shown in Fig. 6. The measured results demonstrate a $-10-\mathrm{dB}$ impedance bandwidth $\left(\left|\mathrm{S}_{11}\right|<-10 \mathrm{~dB}\right)$ from 2.855 to $3.040 \mathrm{GHz}$, i.e., $185 \mathrm{MHz}$, giving $\mathrm{FBW}=6.3 \%$. The measured results are reasonably well matched to their simulated values. The apparent small discrepancies arose from fabrication tolerances, which in turn lead to an inaccurate coupling strength between the two cavities, e.g., the introduction of an unavoidable tiny air gap (even with the tightening screws) between the two substrates during the manual assembly process leads to a slightly wider bandwidth but at the cost of a slightly poorer roll-off-rate.

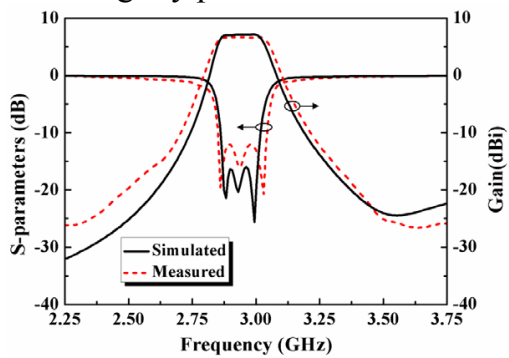

Fig. 6 Comparison of the measured and simulated reflection coefficients and the corresponding realized gains of the dual-cavity SIW-based filtenna.
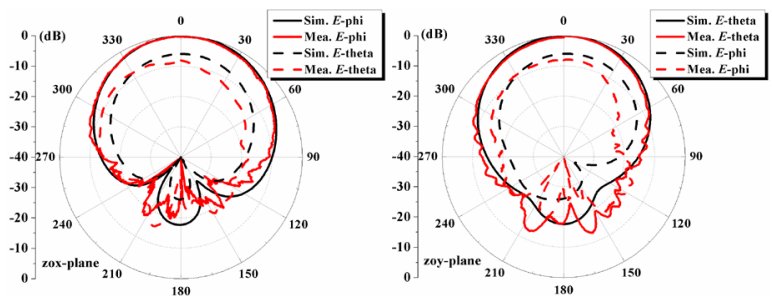

Fig. 7 Simulated and measured radiation patterns of the dual-cavity SIW-based filtenna at $2.93 \mathrm{GHz}$.

The measured and simulated realized gains values at boresight are also shown in Fig. 6. The maximum measured (simulated) realized gain, $6.73(7.18) \mathrm{dBi}$, was obtained in the passband. A very good bandpass filter response with high out-of-band selectivity was achieved in good agreement with the simulations. As discussed in Section III, the radiation patterns are almost uniform over the passband. Fig. 7 shows the simulated and measured radiation patterns at $2.93 \mathrm{GHz}$. Even though the cross-polarization levels are relatively high, which is a consequence of the asymmetry of the currents on the CSRR structure along the $y$-axis as shown in Figs. 5 (a), (c) and (e); this characteristic is advantageous for some special applications such as indoor wireless communications since it leads to better transmission capabilities in rich multipath environments [12].

Table II provides a comparison of the recently reported filtenna designs in [7]-[10] and the one reported herein. Compared to the filtennas with two cavities introduced in [7] and [8], the prototype achieves a comparable peak gain value. It clearly has a better out-of-band selectivity and only one third (one fifth) of the vertical profile and with more than four (two) times the bandwidth reported in [7] ([8]). Compared to the three-cavity [9] and four-cavity [10] designs, it provides a comparable fractional bandwidth with only two cavities and with less than a tenth (a third) of the height in [9] ([10]) and comparable selectivity with [10], but with nearly one half less transverse size and seven times less volume. Moreover, the simulated radiation efficiency values of the proposed design, being influenced mainly by the loss tangent of the substrate, are higher than $80 \%$ over the entire operational frequency range.

TABLE II. COMPARISONS OF THE DESIGN AND PERFORMANCE OF RECENTLY REPORTED FILTENNAS AND THE DUAL-CAVITY SIW-BASED FILTENNA

\begin{tabular}{|c|c|c|c|c|c|c|c|}
\hline Ref. & $\begin{array}{c}\text { Coupling } \\
\text { type }\end{array}$ & $\begin{array}{c}\text { No. of } \\
\text { cavities }\end{array}$ & $\begin{array}{c}\text { Thicknes } \\
\mathrm{s} \\
\left(\lambda_{0}\right)\end{array}$ & $\begin{array}{c}\text { Size }\left(\lambda_{0}\right) \text { and } \\
\text { Volume }\left(\lambda_{0}{ }^{3}\right)\end{array}$ & $\begin{array}{c}\text { *Lower/ } \\
\text { Upper } \\
\text { Selectivity } \\
(\mathrm{dB} / \mathrm{GHz})\end{array}$ & $\begin{array}{c}\text { Real. } \\
\text { Gain } \\
(\mathrm{dBi})\end{array}$ & $\begin{array}{c}\text { FBW } \\
(\%)\end{array}$ \\
\hline$[7]$ & Slot & 2 & 0.08 & $\begin{array}{c}0.99 \times 0.83 \times 0.08 \\
0.0657\end{array}$ & $26 / 57$ & 6.79 & 1.56 \\
\hline$[8]$ & Slot & 2 & 0.15 & $\begin{array}{c}0.49 \times 0.37 \times 0.15 \\
0.0272\end{array}$ & $63 / 28$ & 6.58 & 3 \\
\hline$[9]$ & Slot & 3 & 0.33 & $\begin{array}{c}0.59 \times 0.47 \times 0.33 \\
0.0915\end{array}$ & $96 / 55$ & 6 & 5.5 \\
\hline$[10]$ & Window & 4 & 0.1 & $\begin{array}{c}0.93 \times 0.85 \times 0.1 \\
0.0791\end{array}$ & $113 / 74$ & 6.1 & 6 \\
\hline $\begin{array}{c}\text { This } \\
\text { work }\end{array}$ & Probe & 2 & 0.03 & $\begin{array}{c}0.62 \times 0.62 \times 0.03 \\
0.0115\end{array}$ & $103 / 85$ & 6.73 & 6.3 \\
\hline
\end{tabular}

*Each selectivity value is calculated as $(20 \mathrm{~dB}-3 \mathrm{~dB}) / / f_{20 \mathrm{~dB}}-f_{3 \mathrm{~dB}}$, , where $f_{20 \mathrm{~dB}}$ and $f_{3 \mathrm{~dB}}$ are, respectively, the frequency points corresponding to the decrease from the maximum realized gain by $20 \mathrm{~dB}$ and $3 \mathrm{~dB}$ [13].

\section{CONCLUSION}

A dual-cavity SIW-based filtenna with low profile, compact size, and enhanced bandwidth was reported in this letter. Its design and radiation characteristics were compared with recently reported filtennas. Its compact size and enhanced bandwidth advantages were facilitated. A CSRR-modified patch was employed as the radiator rather than cavity-backed rectangular slots. Energy coupling between the cavities was facilitated by a conducting post rather than by additional slots. Because the post connected the ground and top surfaces, a third resonance arising from the CSRR-modified patch radiator was tuned to overlap with the two resonances associated with the dual-cavity filter. Because the realized dual-cavity filtenna offers the additional advantages of low cost, low profile, and easy integration with other planar circuits, its advantageous design and performance characteristics make it very suitable for practical applications in many compact, wireless mobile platforms. 


\section{REFERENCES}

[1] G. Q. Luo, Z. F. Hu, Yin, L. X. Dong, and L. L. Sun, "Planar slot antenna backed by substrate integrated waveguide cavity," IEEE Antennas Wireless Propag. Lett., vol. 7, pp. 236-239, 2008.

[2] S. Yun, D. Y. Kim, and S. Nam, "Bandwidth enhancement of cavity-backed slot antenna using a via-hole above the slot," IEEE Antennas Wireless Propag. Lett., vol. 11, pp. 1092-1095, 2012.

[3] G. Q. Luo, Z. F. Hu, W. J. Li, X. H. Zhang, L. L. Sun, and J. F. Zheng, "Bandwidth-enhanced low-profile cavity-backed slot antenna by using hybrid SIW cavity modes," IEEE Trans. Antennas Propag., vol. 60, no. 4, pp. 1698-1704, Apr. 2012.

[4] M. Mbaye, J. Hautcoeur, L. Talbi, and K. Hettak, "Bandwidth broadening of dual-slot antenna using substrate integrated waveguide (SIW)," IEEE Antennas Wireless Propag. Lett., vol. 12, pp. 1169-1171, 2013.

[5] S. Mukherjee, A. Biswas, and K. V. Srivastava, "Broadband substrate integrated waveguide cavity-backed bow-tie slot antenna," IEEE Antennas Wireless Propag. Lett., vol. 13, pp. 1152-1155, 2014.

[6] T. Zhang, W. Hong, Y. Zhang, and K. Wu, "Design and analysis of SIW cavity backed dual-band antennas with a dual-mode triangular-ring slot," IEEE Trans. Antennas Propag., vol. 62, no. 10, pp. 5007-5016, Oct. 2014.

[7] H. Chu, C. Jin, J. X. Chen, and Y. X. Guo, "A 3-D millimeter-wave filtering antenna with high selectivity and low cross-polarization," IEEE Trans. Antennas Propag., vol. 63, no. 5, pp. 2375-2380, May 2015.

[8] Y. Yusuf, H. T. Cheng, and X. Gong, "A seamless integration of 3-D vertical filters with highly efficient slot antennas," IEEE Trans. Antennas Propag., vol. 59, no. 11, pp. 4016-4022, Nov. 2011.

[9] H. T. Cheng, Y. Yusuf, and X. Gong, "Vertically integrated three-pole filter/antennas for array applications," IEEE Antennas Wireless Propag. Lett., vol. 10, pp. 278-281, Apr. 2011.

[10] Y. Yusuf and X. Gong, "Compact low-loss integration of high-Q 3-D filters and highly efficient slot antennas," IEEE Trans. Microw. Theory Tech., vol. 59, no. 4, pp. 857-865, Apr. 2011.

[11] J. S. Hong and M. J. Lancaster, Microstrip Filter for RF/Microwave Applications. New York, NY, USA: Wiley, 2001.

[12] K.-L Wong, Compact and Broadband Microstrip Antennas, New York: Wiley, ch. 3, 2002.

[13] F. -C. Chen, R. -S. Li, J. -M. Qiu and Q. -X. Chu, "Sharp-rejection wideband bandstop filter using stepped impedance resonators, " IEEE Trans. Compon., Packag., Manuf. Technol., vol. 7, no. 3, pp. 444-449, Mar. 2017. 